\title{
ACTIVIDAD DE GLUTATIÓN PEROXIDASA EN BOVINOS LECHEROS A PASTOREO CORRELACIONADA CON LA CONCENTRACIÓN SANGUINEA Y PLASMÁTICA DE SELENIO'
}

\author{
ALEJANDRO CEBALLOS ${ }^{2}$, FERNANDO GERMAN WITTWER ${ }^{3}$, PEDRO ALBERTO CONTRERAS ${ }^{4}$, \\ EDUARDO QUIROZ ${ }^{5}$ y HELGA LIDIA BÖHMWALD ${ }^{6}$
}

\begin{abstract}
RESUMEN - Con el objeto de validar una técnica para determinar la actividad sanguínea de glutatión peroxidasa (GSH-Px; EC 1.11.1.9) en el Laboratorio de Patología Clínica de la Universidad Austral de Chile y establecer la correlación entre su actividad y la concentración sanguínea y plasmática de selenio (Se) en bovinos a pastoreo en rebaños lecheros del sur de Chile, se tomaron 5-10 mL de sangre heparinizada a 112 vacas de ocho rebaños en la provincia de Valdivia. La actividad enzimática se analizó mediante una técnica cinética, y el Se por activación de neutrones. Fueron calculadas la inexactitud e imprecisión de la técnica cinética y se describen el rango, promedio y desviación estándar de la actividad enzimática. La correlación entre la actividad sanguínea de GSH-Px y la concentración de Se fue obtenida mediante el coeficiente de correlación simple. La inexactitud e imprecisión fueron 5,9\% y $10 \%$, respectivamente. La actividad de GSH-Px fue $89 \pm 45 \mathrm{U} / \mathrm{g}$ de hemoglobina $(\mathrm{Hb})$ y la correlación entre las variables señaladas fue $\mathrm{r}=0,97(\mathrm{P}<0,05)$. Según estos resultados, es posible recomendar el uso rutinario de la técnica descrita. La correlación señalada permite anotar que en bovinos a pastoreo la actividad de GSH-Px está relacionada con la concentración sanguínea de selenio.
\end{abstract}

Términos para índice: antioxidantes, estrés oxidativo, minerales.

\section{BLOOD ACTIVITY OF GLUTATHIONE PEROXIDASE AND ITS CORRELATION WITH BLOOD SELENIUM CONCENTRATION IN GRAZING DAIRY CATTLE}

ABSTRACT - Selenium (Se) is part of the antioxidant enzyme glutathione peroxidase (GSH-Px; EC 1.11.1.9) structure, whose blood activity is related to the blood level of selenium. This study was designed to validate the analytical method to analyze the GSH-Px blood activity at the Clinical Pathology Laboratory of the Universidad Austral de Chile, and to correlate it with blood Se level in dairy cattle from the South of Chile. Blood heparinized samples were taken from 112 dairy cows from eight dairy herds located at Valdivia province, Chile. A kinetic NADPH-dependent technique was used to analyze the blood GSH-Px, and the content of Se in blood and plasma was analyzed by neutron activation. The Se concentration in blood was analyzed in 12 samples to correlate GSH-Px blood activity with blood and plasma Se level. The inaccuracy and imprecision were $5.9 \%$ and $10 \%$, respectively. The mean and standard deviation of the obtained values were $89 \pm 45 \mathrm{U} / \mathrm{g}$ of hemoglobin $(\mathrm{Hb})$. The correlation between blood selenium and blood GSH-Px activity was $\mathrm{r}=0.97(\mathrm{P}<0.05)$. Accordingly, it is possible to analyze the blood activity of GSH-Px by using the described technique. The blood activity of GSH-Px was related to the blood selenium level.

Index terms: antioxidants, oxidative stress, minerals.

${ }^{1}$ Aceptado para publicación en 26 de noviembre. Proyecto FONDECYT-Chile 1951062.

${ }^{2}$ Méd. Vet. Zoot., M.Sc., Instituto de Ciencias Clínicas Veterinarias, Universidad Austral de Chile, Casilla 567, Valdivia, Chile.E-mail: aceballo@uctem.cl

${ }^{3}$ Méd. Vet., M.Sc., Instituto de Ciencias Clínicas Veterinarias, Universidad Austral de Chile, Valdivia, Chile. E-mail: fwittwer@uach.cl

\footnotetext{
${ }^{4}$ Méd. Vet., M.Phil., Instituto de Ciencias Clínicas Veterinarias, Universidad Austral de Chile, Valdivia, Chile. E-mail: pcontre1@uach.cl

${ }^{5}$ Quím., Instituto de Química, Universidad Austral de Chile, Valdivia,Chile.E-mail: rquiroz@uach.cl

${ }^{6}$ Tec. Méd., Instituto de Ciencias Clínicas Veterinarias, Universidad Austral de Chile, Valdivia, Chile.
}

Pesq. agropec. bras., Brasília, v.34, n.12, p.2331-2338, dez. 1999 


\section{INTRODUCCIÓN}

El estrés oxidativo produce reacciones que alteran la permeabilidad de las membranas celulares, la funcionalidad enzimática y el tono muscular (Miller et al., 1993). También ha sido asociado con la presentación de algunas patologías en el bovino como la enfermedad del músculo blanco, enfermedades metabólicas, infertilidad e inmunosupresión (Foucras et al., 1996; Lomba, 1996).

La glutatión peroxidasa (GSH-Px; EC 1.11.1.9) es una metaloenzima que forma parte del sistema glutatión, señalado como el principal sistema antioxidante en el organismo. Se han identificado tres tipos de GSH-Px: celular, extracelular (plasmática) y fosfolípido hidroperóxido glutatión peroxidasa. La estructura de la enzima celular está conformada por cuatro subunidades de $22 \mathrm{Kdaltons}$ cada una, donde cada subunidad contiene un residuo de selenocisteína (Burk \& Hill, 1993).

Las funciones de la GSH-Px son: inactivar algunos de los radicales libres derivados del oxígeno que se forman en el organismo como consecuencia del metabolismo aerobio; así, esta enzima es responsable de la protección de la membrana de las células que funcionan en un presencia de oxígeno (Miller et al., 1993), intervenir en la cascada de reacciones que catalizan la formación de prostaglandinas, leukotrienos, prostaciclinas y tromboxanos a partir del ácido araquidónico (Stadtman, 1990), se relaciona con el normal funcionamiento del sistema inmunológico (Cao et al., 1992) y con la integridad funcional del tracto reproductivo tanto en machos como en hembras (Hurley \& Doane, 1989).

La presencia de selenio (Se) en la estructura de la enzima permite que exista una alta relación entre la concentración sanguínea y tisular de Se con la actividad de GSH-Px; además, la función metabólica conocida del Se es formar parte de la estructura de la enzima. En bovinos la correlación entre la actividad enzimática en los eritrocitos y la concentración sanguínea de Se es alta; así, se han descrito valores de correlación que varían entre 0,67 y 0,97 (Backall \& Scholz, 1979; Scholz et al., 1981; Van Saun et al., 1989). No obstante, los componentes celulares de la sangre contienen la mayor proporción de Se; así, la correlación entre la actividad de la enzima y la concentración plasmática de Se es baja (Scholz \& Hutchinson, 1979). En otras especies también se ha descrito una alta correlación entre la actividad de GSH-Px y la concentración de Se en la sangre; así, se ha señalado un $\mathrm{r}=0,94(\mathrm{P}<0,001)$ en equinos (Maylin et al., 1980).

En consideración a lo anterior, este estudio tiene por objeto validar una técnica analítica para determinar la actividad sanguínea de glutatión peroxidasa, y también establecer la correlación entre la actividad sanguínea de GSH-Px y la concentración de Se en sangre en bovinos a pastoreo de rebaños lecheros en el sur de Chile, y entre la actividad enzimática y el contenido de Se en el forraje.

\section{MATERIAL Y METODOS}

En la X Región de Chile ( $39^{\circ} \mathrm{LS}$ y $\left.73^{\circ} \mathrm{LO}\right)$ se seleccionaron ocho predios lecheros según su masa ganadera, tipo de alimentación del rebaño y que los animales no estuvieran recibiendo una suplementación con Se adicional al consumido en la pradera, el concentrado o la sal mineralizada.

\section{Toma de muestras}

Se seleccionó por cada predio dos grupos de siete animales mantenidos en pastoreo, a los que se tomaron $5-10 \mathrm{~mL}$ de sangre heparinizada mediante venopunción coccígea empleando el sistema de tubos al vacío. Los tubos fueron tratados previamente con una solución de ácido nítrico $\left(\mathrm{HNO}_{3}\right)$ al $15 \%$.

Las muestras se llevaron dentro de 24 horas al Laboratorio de Patología Clínica Veterinaria de la Universidad Austral de Chile, donde se determinó la concentración de hemoglobina mediante el método de la cianometahemoglobina (Jain, 1986). Posteriormente fueron hemolizados $50 \mu \mathrm{L}$ de sangre en $2 \mathrm{~mL}$ de una solución diluyente proporcionada por el fabricante del reactivo, conservándose el hemolizado a $-25^{\circ} \mathrm{C}$ en envases plásticos debidamente identificados hasta su posterior análisis.

Asimismo, se tomaron muestras de forraje de las praderas donde pastoreaban los animales seleccionados para determinar la concentración de Se y correlacionarla con la actividad de GSH-Px en sangre y plasma.

Posteriormente, se seleccionaron 12 muestras de las 112 obtenidas inicialmente según su actividad de GSH-Px (baja, media, alta) para determinar la concentración de $\mathrm{Se}$ en la sangre y el plasma, y correlacionarla con la actividad de GSH-Px. Las muestras seleccionadas, al igual que las 
de forraje, fueron enviadas al laboratorio de la Comisión Chilena de Energía Nuclear donde se analizó la concentración de Se.

\section{Método analítico}

La actividad sanguínea de GSH-Px se analizó mediante un reactivo comercial basado en una técnica cinética compuesta NADPH-dependiente (Paglia \& Valentine, 1967). Los análisis se realizaron en un fotómetro semiautomático Hitachi 4020 con un conjunto múltiple de fotodiodos de diferente longitud de onda.

La técnica está basada en la determinación de la oxidación del glutatión reducido (GSH) por el peróxido de hidrógeno $\left(\mathrm{H}_{2} \mathrm{O}_{2}\right)$ en una reacción catalizada por la GSH-Px que contiene el hemolizado (Reacción 1). El GSH se mantiene a una concentración constante durante la reacción mediante la adición de glutatión reductasa (GR; EC 1.6.4.2) y fosfato de nicotinamida adenindinucleótido (NADPH); así, el glutatión oxidado (GSSG) se reduce formando GSH y el NADPH es oxidado y consumido durante la reacción (Reacción 2).

$2 \mathrm{GSH}+\mathrm{H}_{2} \mathrm{O}_{2}-\ldots-\mathrm{GSSG}+2 \mathrm{H}_{2} \mathrm{O}$ (Reacción 1)

GSSG + NADPH - - - - - - 2 GSH + NADP (Reacción 2)

La tasa de formación de GSH es monitoreada mediante la disminución en la absorbancia a $340 \mathrm{~nm}$ producida por el consumo del NADPH a $37^{\circ} \mathrm{C}$. La diferencia en el consumo de NADPH entre el blanco y la muestra determina la actividad enzimática de GSH-Px, siendo ésta directamente proporcional a la disminución en la absorbancia (Langlands et al., 1980). La composición de los reactivos y la concentración final en el ensayo se describen en el Cuadro 1.

En un tubo de reacción se mezclaron $500 \mu \mathrm{L}$ del Reactivo $\mathrm{N}^{\circ} 1$ con $10 \mu \mathrm{L}$ de agua destilada (blanco) ó $10 \mu \mathrm{L}$ del hemolizado (muestra) y se incubó a $37^{\circ} \mathrm{C} \mathrm{du}-$ rante 5 minutos; posteriormente, se agregaron $20 \mu \mathrm{L}$ de la solución con el cumene y se leyó en el fotómetro la disminución en la absorbancia durante 2 minutos a una longitud de onda de $340 \mathrm{~nm}$ y una temperatura de $37^{\circ} \mathrm{C}$.

La actividad de GSH-Px en U/litro de hemolizado se obtuvo de la ecuación:

$\mathrm{U} / \mathrm{L}=\left(8412 \times \Delta \mathrm{Abs}_{\min .}\right.$ muestra $)-\left(8412 \times \Delta \mathrm{Abs}_{\min .}\right.$. blanco $)$

Para calcular la actividad de GSH-Px de la muestra en $\mathrm{U} / \mathrm{g}$ de hemoglobina $(\mathrm{Hb})$ se emplea la ecuación:

$\mathrm{U} / \mathrm{g} \mathrm{Hb}=(\mathrm{U} / \mathrm{L}$ hemolizado $\mathrm{x} 41) /$ hemoglobina $(\mathrm{g} / \mathrm{L})$.

La exactitud de la técnica se controló mediante el análisis de un hemolizado control con una actividad enzimática conocida y cuyo valor promedio era $539 \mathrm{U} / \mathrm{L}$ de hemolizado. También se realizó un control externo comparando el valor obtenido para la actividad enzimática con el valor obtenido en el Laboratorio de la Clínica de Enfermedades del Bovino de la Escuela de Medicina Veterinaria de Hannover, Alemania.

Para el análisis de la precisión se determinó 20 veces la actividad enzimática en un hemolizado preparado en el Laboratorio y obtenido de una muestra de sangre tomada a un animal clínicamente sano.

El Se, tanto en sangre, plasma y forraje, fue determinado mediante activación de neutrones; en esta técnica la muestra es sometida a irradiación marcándose después con un isótopo estable y determinando la cantidad producida del elemento (Gissel-Nielsen et al., 1984).

\section{Análisis de los resultados}

Se analizaron la exactitud, inexactitud, precisión e imprecisión de la técnica analítica para la determinación de la actividad sanguínea de GSH-Px (Kohlschein, 1993). Los resultados se describen mediante la obtención del rango de actividad y el cálculo de las estimadas promedio (x) y desviación estándar (DE) (Domènech, 1980). Se obtuvo la frecuencia de grupos de animales con valores bajo $130 \mathrm{U} / \mathrm{g} \mathrm{Hb}$, valor que ha sido considerado como el límite bajo el cual habría una deficiencia marginal de Se (Ceballos $\&$ Wittwer, 1996). Igualmente, se obtuvo la frecuencia de muestras de forraje con un contenido de Se inferior a $0,1 \mathrm{ppm}$, valor señalado como la concentración mínima requerida en el forraje para bovinos a pastoreo (McDowell, 1992).

Las correlaciones entre la actividad sanguínea de GSH-Px y la concentración de Se en la sangre, el plasma $\mathrm{y}$ el forraje fueron establecidas mediante un coeficiente de correlación simple (Domènech, 1980).

\section{CUADRO 1. Composición de los reactivos y concen- tración final en el ensayo para la deter- minación de la actividad sanguínea de glutatión peroxidasa ${ }^{1}$.}

\begin{tabular}{lc}
\hline Componente & $\begin{array}{c}\text { Concentración } \\
\text { en el ensayo }\end{array}$ \\
\hline Reactivo No 1: & \\
$\quad$ Glutatión reducido & $4 \mathrm{mmol} / \mathrm{L}$ \\
$\quad$ Glutatión reductasa (EC 1.6.4.2) & $>0,5 \mathrm{U} / \mathrm{L}$ \\
NADPH & $0,28 \mathrm{mmol} / \mathrm{L}$ \\
Solución buffer: & \\
$\quad$ Buffer fosfato (pH 7,2) & $0,05 \mathrm{~mol} / \mathrm{L}$ \\
$\quad$ EDTA (sal tetrasódica) & $4,3 \mathrm{mmol} / \mathrm{L}$ \\
Cumene hidroperóxido & $0,18 \mathrm{mmol} / \mathrm{L}$ \\
\hline
\end{tabular}

${ }^{1}$ Adaptado de Randox Laboratories (1994). 


\section{RESULTADOS Y DISCUSIÓN}

El promedio y la DE para la actividad de GSH-Px en el hemolizado control fue $529 \pm 37$ U/L de hemolizado, con un coeficiente de variación igual al 7\% (Fig. 1). La inexactitud de la técnica fue de $32 \mathrm{U} / \mathrm{L}$ de hemolizado, lo que corresponde a un $5,9 \%$.

En el hemolizado preparado en el laboratorio se encontró una actividad enzimática de $274 \pm 28 \mathrm{U} / \mathrm{L}$, observándose un coeficiente de variación del $10 \%$. La imprecisión fue $28 \mathrm{U} / \mathrm{L}$, lo que corresponde al $10 \%$ de error esperable en análisis posteriores, teniendo una posibilidad del $90 \%$ de obtener valores entre 219 y $330 \mathrm{U} / \mathrm{L}$ al repetir 100 veces el análisis del hemolizado preparado en el Laboratorio.

En las muestras, el promedio y la DE para la actividad sanguínea de GSH-Px en los 10 grupos de animales fue $89 \pm 45 \mathrm{U} / \mathrm{g} \mathrm{Hb}$, con un rango entre 3 y $371 \mathrm{U} / \mathrm{g} \mathrm{Hb}$, encontrándose en todos los grupos valores promedio inferiores a $130 \mathrm{U} / \mathrm{g} \mathrm{Hb}$, valor señalado como indicador de una deficiencia marginal de Se.

En el Cuadro 2 se describen el promedio y la DE para la concentración sanguínea y plasmática de Se en las 12 muestras seleccionadas, así como el contenido de Se en las 10 muestras de forraje.

En las muestras de forraje se encontró en 6 de las 10 muestras analizadas contenidos de Se bajo $0,1 \mathrm{ppm}$, valor señalado como indicador de una baja concentración de Se para bovinos en pastoreo.

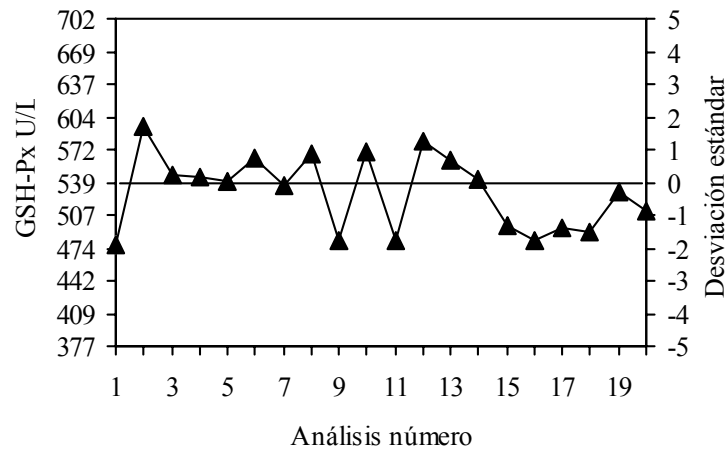

FIG. 1. Gráfica de Levey-Jennings del análisis de la actividad sanguínea de glutatión peroxidasa (GSH-Px) en un hemolizado control (Ransel control, lote 099 RS. Randox Laboratories, 1994).
La correlación que se encontró para la actividad de GSH-Px y la concentración de Se en sangre (Fig. 2) fue $\mathrm{r}=0,97(\mathrm{P}<0,05)$. Para la correlación entre la actividad enzimática y la concentración de Se en el plasma (Fig. 3) se observó un $\mathrm{r}=0,89$ $(\mathrm{P}<0,05)$. La correlación entre la actividad de la enzima y el contenido de Se en el forraje (Fig. 4) fue $\mathrm{r}=0,93(\mathrm{P}<0,05)$.

Los valores de la actividad enzimática que se obtuvieron al analizar el hemolizado control sustrayendo los valores del blanco de reacción, estuvieron dentro del rango señalado como objetivo (474 - $604 \mathrm{U} / \mathrm{L}$ de hemolizado). Asimismo, éstos valores fueron similares a los encontrados en el La-

CUADRO 2. Promedio, desviación estándar y rango de la concentración de selenio en sangre y plasma de 12 bovinos a pastoreo, y contenido de selenio en el forraje de $\mathbf{1 0}$ predios en la X Región de Chile.

\begin{tabular}{lcc}
\hline \multirow{2}{*}{ Tejido } & \multicolumn{2}{c}{ Concentración de selenio } \\
\cline { 2 - 3 } & $\mathrm{x} \pm \mathrm{DE}$ & Rango \\
\hline Sangre $(\mu \mathrm{mol} / \mathrm{L})$ & $0,52 \pm 0,36$ & $0,06-1,24$ \\
Plasma $(\mu \mathrm{mol} / \mathrm{L})$ & $0,26 \pm 0,17$ & $0,13-0,67$ \\
Forraje $\left(\mathrm{ppm}^{1}\right)$ & $0,11 \pm 0,05$ & $0,06-0,21$ \\
\hline
\end{tabular}

${ }^{1}$ Base seca.

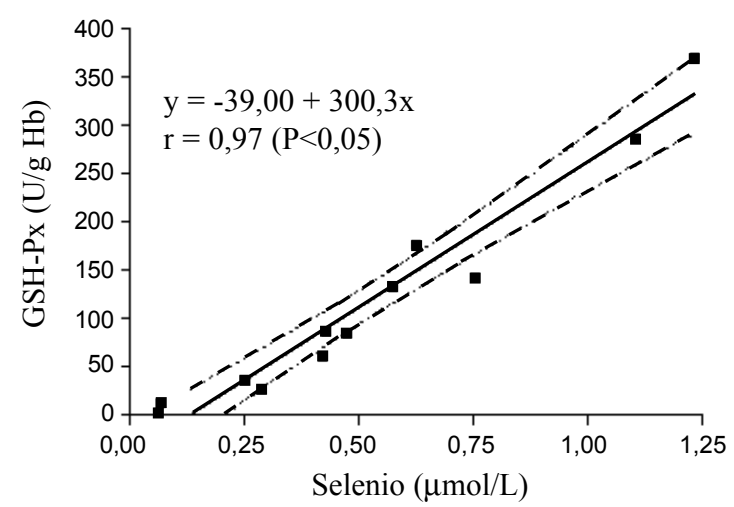

FIG. 2. Regresión y correlación entre la actividad sanguínea de glutatión peroxidasa (GSH-Px) y la concentración de selenio en sangre de 12 bovinos a pastoreo en la $\mathrm{X}$ Región de Chile. 
boratorio de la Escuela de Medicina Veterinaria de Hannover, Alemania, donde se realizó el control externo. Los resultados de ambos Laboratorios estuvieron dentro del objetivo esperado (Fig. 1). La variación observada en las determinaciones fue baja $(5,9 \%)$ con respecto a la actividad enzimática promedio en el hemolizado control (539 U/L). Por lo anterior, es posible señalar que el método analítico utilizado es exacto, ya que la exactitud de una técnica está dada cuando se obtienen resultados cercanos a un valor objetivo al analizar un estándar con valores conocidos (Kohlschein, 1993).

El coeficiente de variación obtenido al analizar la actividad enzimática en un hemolizado preparado en el Laboratorio fue un $10 \%$. En otros estudios se han señalado variaciones que fluctúan entre 1,8\% y $15 \%$ (Wilson \& Judson, 1976; Davidson et al., 1990). No obstante, cabe señalar que en el método analítico empleado en los reportes descritos hay variaciones en el $\mathrm{pH}$, temperatura, concentración de la coenzima y concentración del sustrato con respecto a la técnica utilizada en este estudio.

Se ha señalado que una técnica analítica es precisa cuando al analizar repetidamente una misma muestra, la variación entre los valores obtenidos es baja. Por lo general el coeficiente de variación o imprecisión de la técnica no debe ser superior al 10\%, excepto para las determinaciones enzimáticas (Me-

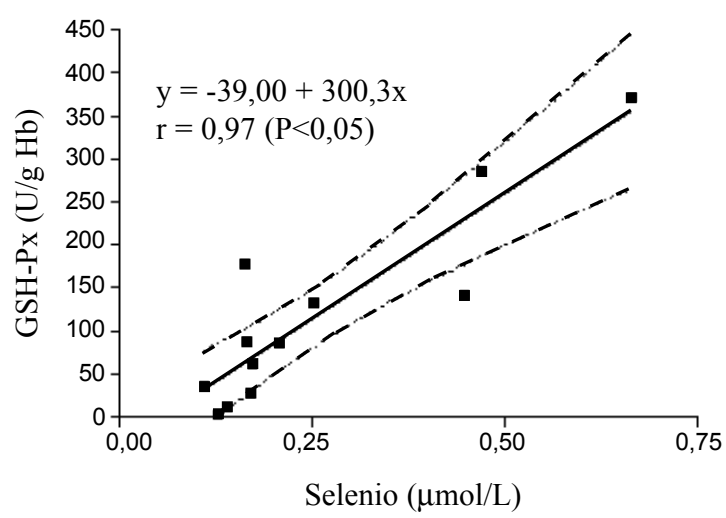

FIG. 3. Regresión y correlación entre la actividad sanguínea de glutatión peroxidasa (GSH-Px) y la concentración plasmática de selenio de 12 bovinos a pastoreo en la $X$ Región de Chile. rino, 1977); por lo tanto, es posible señalar que la técnica analítica empleada en este estudio es precisa.

Se observaron los 10 grupos de animales con una actividad enzimática promedio inferior a $130 \mathrm{U} / \mathrm{g} \mathrm{Hb}$. En otros estudios también se ha encontrado que los animales mantenidos en pastoreo presentan una baja actividad enzimática de GSH-Px en sangre (Backall \& Scholz, 1979; Calamari et al., 1989).

En bovinos la correlación entre la actividad de GSH-Px en sangre y la concentración sanguínea de Se es alta, lo que concuerda con los resultados observados en este estudio (Fig. 2). En diferentes estudios se ha encontrado una alta correlación entre la concentración de Se en la sangre y la actividad de GSH-Px; así, se ha observado un $\mathrm{r}=0,93(\mathrm{P}<0,0001)$ (Erskine et al., 1987) y un $\mathrm{r}=0,67(\mathrm{P}<0,0001)$ (Van Saun et al., 1989). Más recientemente se ha encontrado una correlación entre 0,78 y 0,95 para la asociación entre las variables descritas (Kessler et al., 1992; Jukola, 1994). Se ha señalado en bovinos mantenidos en pastoreo en áreas con diferentes concentraciones de Se una correlación de $\mathrm{r}=0,95$ (Stevens et al., 1985).

La correlación entre la actividad enzimática y la concentración plasmática del mineral fue $\mathrm{r}=0,89$ $(\mathrm{P}<0,05)$ (Fig. 3), observándose que el contenido de

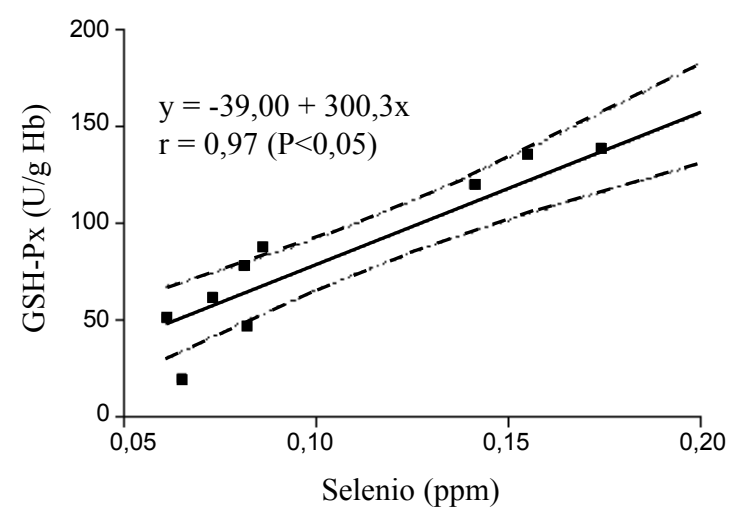

FIG. 4. Regresión y correlación entre la actividad sanguínea promedio de glutatión peroxidasa (GSH-Px) en 10 grupos de bovinos a pastoreo en la $X$ Región de Chile y el contenido de selenio en el forraje.

Pesq. agropec. bras., Brasília, v.34, n.12, p.2331-2338, dez. 1999 
Se en el plasma representaba el $45 \%$ de la concentración sanguínea. Lo anterior contrasta con lo indicado por Scholz \& Hutchinson (1979), quienes anotan que los componentes celulares de la sangre contienen la mayor proporción de Se; así, la correlación entre la actividad de la enzima y la concentración plasmática de Se es baja.

Los resultados observados en este estudio permiten corroborar la existencia de una relación entre la concentración sanguínea y plasmática de Se y la actividad de GSH-Px en el mismo tejido. Por lo tanto, siendo esta concentración de Se en sangre altamente dependiente del consumo del mineral en la ración, la actividad sanguínea de la enzima será o no la adecuada según el contenido del mineral en la dieta.

El rango de la concentración de selenio en las muestras de pasto de los 10 predios fue 0,061 - 0,213 ppm, observándose en 6 de los 10 predios valores inferiores a $0,1 \mathrm{ppm}$.

La concentración de Se que se ha señalado para forrajes es variable, siendo la concentración y la disponibilidad del mineral en el suelo, los principales factores que influyen en el contenido del elemento en la planta. Por lo tanto, cualquier fenómeno que induzca modificaciones en el contenido de Se en el suelo, será una fuente de variación para la concentración del mineral en la pradera (McDowell, 1992). El origen y tipo de suelo, la utilización de fertilizantes, las condiciones climáticas, el pH y la naturaleza química de los compuestos de Se disponibles, son algunos de los factores que pueden producir variaciones en el contenido de Se en el forraje (GisselNielsen et al., 1984). La baja concentración de Se que se ha encontrado en algunas praderas de los predios seleccionados, estaría dada por los factores edáficos y climáticos que caracterizan la X Región en Chile.

Smith et al. (1974) han señalado que un aumento en el consumo de Se en la ración produce un aumento en la actividad sanguínea de GSH-Px y que su correlación con el contenido del mineral en la dieta era superior a $0,70(\mathrm{P}<0,001)$. Recientemente se ha señalado que la correlación entre el contenido de Se la ración y la actividad enzimática es moderada, reportándose un $\mathrm{r}=0,73$ (Weiss et al., 1990). La asociación entre estas variables estaría dada por la relación suelo-planta-animal, ya que el contenido de Se en el suelo y su disponibilidad para las plantas, determinarán la concentración sanguínea del mineral en animales a pastoreo (Langlands et al., 1981).

El animal está en capacidad de utilizar el Se que le aporta el forraje para satisfacer su requerimiento; por lo tanto, a partir de esta fuente nutricional se puede obtener el Se para desarrollar la función biológica conocida, como es la incorporación en la estructura de GSH-Px (Underwood, 1981).

En este estudio se demostró la correlación que existe entre la actividad de GSH-Px con la concentración de Se en sangre y plasma (Figs. 2 y 3); por lo tanto, siendo la actividad enzimática dependiente de la concentración de Se en sangre, esta actividad dependería de la fuente a partir de la cual se obtiene el Se. La única fuente de Se para los animales seleccionados en este estudio era el forraje; así, siendo la pradera la única fuente nutricional, la actividad sanguínea de GSH-Px igualmente es dependiente del mismo alimento.

Lo anterior queda demostrado con la correlación obtenida entre la actividad sanguínea de GSH-Px en animales a pastoreo y el contenido de Se en el forraje (Fig. 4).

La determinación de la actividad enzimática de GSH-Px ha sido utilizada con éxito en la Región para conocer el balance metabólico nutricional de Se en bovinos lecheros a pastoreo, así se han logrado identificar deficiencias nutricionales de Se en el sur de Chile (Ceballos et al., 1998). Además, esta técnica permite en una forma más económica establecer el balance de Se en los bovinos, ofreciendo así una ventaja sobre otras determinaciones que pueden resultar más costosas.

\section{CONCLUSIONES}

1. La técnica descrita para el análisis de la actividad sanguínea de GSH-Px posee la exactitud y precisión adecuadas para implementarla y usarla en forma rutinaria.

2. La actividad sanguínea de GSH-Px en bovinos lecheros mantenidos en pastoreo está correlacionada con la concentración de Se tanto en la sangre y el plasma del animal, como en la prade- 
ra, lo que demuestra que existe una dependencia de la actividad enzimática del aporte de Se a partir de la ración, y confirma la relación planta-animal para este mineral.

\section{REFERENCIAS}

BACKALL, K.A.; SCHOLZ, R.W. Reference values for a field test to estimate inadequate glutathione peroxidase activity and selenium status in the blood of cattle. American Journal of Veterinary, Research, v.40, n.5, p.733-738, May 1979.

BURK, R.F.; HILL, K.E. Regulation of selenoproteins. Annual Review of Nutrition, v.13, p.65-81, 1993.

CALAMARI, L.; BERTONI, G.; MAIANTI, M.G.; CAPPA, V. Sull'utilità di nuovi parametri ematochimici nella valutazione del profilo metabolico delle lattifere. Zootecnica e Nutrizione Animale, v.15, n.3, p.191-210, giug. 1989.

CAO, Y-Z.; MADDOX, J.F.; MASTRO, A.M.; SCHOLZ, R.W.; HILDEBRANDT, G.; REDDY, C.C. Selenium deficiency alters the lipoxygenase pathway and mitogenic response in bovine lymphocytes. Journal of Nutrition, v.122, p.2121-2127, 1992.

CEBAllos, A.; WITTWER, F.G. Metabolismo del selenio en rumiantes. Archivos de Medicina Veterinaria, v.28, n.2, p.5-18, 1996.

CEBALLOS, A.; WITTWER, F.G.; CONTRERAS, P.A.; BÖHMWALD, H. Actividad sanguínea de glutatión peroxidasa en rebaños lecheros a pastoreo: variación según edad y época del año. Archivos de Medicina Veterinaria, v.30, n.1, p.13-22, 1998.

DAVIDSON, W.B.; KENNEDY, D.G.; HUGHES, P.J.; BLANCHFLOWER, W.J. The stability of glutathione peroxidase activity in plasma form cattle, pigs and sheep on storage in the presence and absence of glutathione. Veterinary Research Communications, v.14, p.441-446, 1990.

DOMÈNECH, J.M. Bioestadística: métodos estadísticos para investigadores. Barcelona: Herder, 1980. 642p.

ERSKINE, R.J.; EBERHART, R.J.; HUTCHINSON, L.J.; SCHOLZ, R.W. Blood selenium concentrations and glutathione peroxidase activities in dairy herds with high and low somatic cell counts. Journal of the American Veterinary Medical Association, v.190, n.11,p.1417-1421, 1987.
FOUCRAS, G.; SCHELCHER, F.; VALARCHER, J.-F.; ESPINASSE, J. La dystrophie musculaire nutritionelle chez les ruminants. Le Point Veterinaire, v.27, n.172, p.33-38, Jan. 1996.

GISSEL-NIELSEN, G.; GUPTA, U.C.; LAMAND, M.; WESTERMARCK, T. Selenium in soils and its importance in livestock and human nutrition. Advances in Agronomy, v.37, p.397-460, 1984.

HURLEY, W.L.; DOANE, R.M. Recent developments in the roles of vitamins and minerals in reproduction. Journal of Dairy Science, v.72, n.3, p.784-804, Mar. 1989.

JAIN, N.C. Schalm's veterinary hematology. Philadelphia: Lea \& Fabiger, 1986. 807p.

JUKOLA, E. Selenium, vitamin E, vitamin A and betacarotene status of cattle in Finland, with special reference to epidemiological udder health and reproduction data. Helsinki: College of Veterinary Medicine-Section of Animal Hygiene, 1994. 195p.

KESSLER, J.; FRIESECKE, H.; KUNZ, P. Sélénium et vitamine $\mathrm{E}$ : approvisionnement de la vache laitière durant la période d'alimentation hivernale. Revue Suisse D'Agriculture, v.24, n.2, p.87-91, 1992.

KOHLSCHEIN, J. Qualitätskontrolle im medizinischen Laboratorium von $A$ bis $Z$. Liederbach: Behringwerke AG, 1993. 91p.

LANGLANDS, J.P.; BOWLES, J.E.; SMITH, A.J.; DONALD, G.E. Selenium concentration in the blood of ruminants grazing northern New South Wales. II. Relationship with geological, pedological and other variables. Australian Journal of Agricultural Research, v.32, p.523-533, 1981.

LANGLANDS, J.P.; DONALD, G.E.; BOWLES, J.E.; SMITH, A.J. Rapid spot test for glutathione peroxidase activity: Comparison with spectophotometric procedure and assessment of the test as a measure of selenium in the blood of grazing ruminants. Australian Journal of Agricultural Research, v.31, p.357-367, 1980.

LOMBA, F. Influence des rapports anions-cations et oxydants-antioxydants dans les rations des vaches laitières en période de tarissement sur l'incidence du syndrome du part. Annales de Médecine Véterinaire, v.140, p.109-122, 1996.

MAYLIN, G.A.; RUBIN, D.S.; LEIN, D.H. Selenium and vitamin $\mathrm{E}$ in horses. Cornell Veterinarian, v.70, p.272-289, 1980.

Pesq. agropec. bras., Brasília, v.34, n.12, p.2331-2338, dez. 1999 
McDOWELL, L.R. Minerals in human and animal nutrition. San Diego: Academic, 1992. 524p.

MERINO, N. de. Control de calidad: evaluación del error, la gráfica de control. Analítica, n.14, p.5-6, 1977.

MILLER, J.K.; BRZEZINSKA-SLEBODZINSKA, E.; MADSEN, F.C. Oxidative stress, antioxidants and animal function. Journal of Dairy Science, v.76, n.9, p.2812-2823, Sept. 1993.

PAGLIA, D.E.; VALENTINE, W.N. Studies on the quantitative and qualitative characterization of erythrocyte gluthatione peroxidase. Journal of Laboratory and Clinical Medicine, v.70, n.1, p.158-169, 1967.

RANDOX LABORATORIES. Procedimientos manuales. Crumlin, 1994. 2p.

SCHOLZ, R.W.; HUTCHINSON, L.J. Distribution of glutathione peroxidase activity and selenium in the blood of dairy cows. American Journal of Veterinary Research, v.40, n.2, p.245-249, Feb. 1979.

SCHOLZ, R.W.; TODHUNTER, D.A.; COOK, L.S. Selenium content and glutathione peroxidase activity in tissues of young cattle fed supplemented whole milk diets. American Journal of Veterinary Research, v.42, n.10, p.1718-1723, Oct. 1981.

SMITH, P.J.; TAPPEL, A.L.; CHOW, C.K. Glutathione peroxidase activity as a function of dietary selenomethionine. Nature, v.247, p.392-393, Feb. 1974.

STADTMAN, T.C. Selenium biochemistry. Annual Review of Biochemistry, v.59, p.111-127, 1990.

STEVENS, J.B.; OLSON, W.G.; KRAEMER, R.; ARCHAMBEAU, J. Serum selenium concentrations and glutathione peroxidase activities in cattle grazing forages of various selenium concentrations. American Journal of Veterinary Research, v.46, n.7, p.1556-1560, July 1985.

UNDERWOOD, E.J. The mineral nutrition of livestock. London: Commonwealth Agricultural Bureaux, 1981. 180p.

VAN SAUN, R.J.; HERDT, T.H.; STOWE, H.D. Maternal and fetal selenium concentrations and their interrelationships in dairy cattle. Journal of Nutrition, v.119, p.1128-1137, 1989.

WEISS, W.P.; TOODHUNTER, D.A.; HOGAN, J.S.; SMITH, K.L. Effect of duration of supplementation of selenium and vitamin $\mathrm{E}$ on periparturient dairy cows. Journal of Dairy Science, v.73, n.11, p.3187-3194, Nov. 1990.

WILSON, P.S.; JUDSON, G.J. Glutathione peroxidase activity in bovine and ovine erythrocytes in relation to blood selenium concentration. British Veterinary Journal, v.132, p.428-434, 1976. 